\title{
Suppression of Superconductivity in YBCO/LCMO Superlattices
}

\author{
F. Chen ${ }^{1,2}$, B. Gorshunov ${ }^{1 \dagger}$, G. Cristiani ${ }^{2}$, H.-U. Habermeier ${ }^{2}$, and M. Dressel ${ }^{1 *}$ \\ ${ }^{1}$ 1. Physikalisches Institut, Universität Stuttgart, \\ Pfaffenwaldring 57, D-70550 Stuttgart, Germany \\ ${ }^{2}$ Max-Planck-Institut für Festkörperforschung, Heisenbergstr. 1, D-70569 Stuttgart, Germany
}

(Dated: March 23, 2018)

\begin{abstract}
The competition of superconductivity and magnetism in superlattices composed of alternating $\mathrm{YBa}_{2} \mathrm{Cu}_{3} \mathrm{O}_{7-d}$ and $\mathrm{La}_{0.67} \mathrm{Ca}_{0.33} \mathrm{MnO}_{3}$ thin films is investigated using low-energy optical spectroscopy. The thickness of the superconducting YBCO layers is varied from $30 \mathrm{~nm}$ to $20 \mathrm{~nm}$ while the thickness of the magnetic LCMO layers is kept constant at $20 \mathrm{~nm}$. We clearly observe that the superconducting condensate density in the superconducting state of superlattice is drastically reduced by the magnetic subsystem which may be connected with proximity effects that distort the gap symmetry and thus suppress superconductivity.
\end{abstract}

PACS numbers: 72.25.Mk; 74.78.Bz; 75.70.Cn; 78.66.-w

Since the early 1960s the interplay of superconductivity (SC) and ferromagnetism (FM) has continuously drawn attention [1]. At first glance SC and FM exclude each other because the magnetic exchange field breaks the Cooper pairs. Nevertheless the possible coexistence of both phenomena due to a spatially modulated order parameter was suggested by Fulde, Ferrell, Larkin and Ovchinnikov [2]; the correspondent state was never observed in conventional SC. A good way to study the effect is to bring the nano-sized layers of the two materials into contact and to view the SC-FM interaction on the level of the proximity effects. The exploration of multilayer systems composed of alternating conventional SC and metallic FM layers was subject of recent reviews [3, 4].

In the last years artificially grown superlattices (SLs) consisting of high- $T_{c}$ superconductors and manganese oxides which exhibit the phenomenon of colossal magnetoresistance have attracted increasing interests for various reasons. On one hand, SLs were developed as an important tool to explore the interplay between the two antagonistic SC and FM ground states, and on the other hand the injection of spin-polarized carriers can lead to new SC switching devices 5]. Due to the structural similarity of the two classes of perovskite compounds, it is possible to construct a unique combination of SC cuprate and strongly FM manganese layers. It has been demonstrated by different groups [6, [7, 8, 9] that although SC and FM are preserved in each subsystem, the superconducting transition temperature $T_{c}$ and the Curie temperature of the magnetic ordering $T_{\text {mag }}$ are considerably suppressed. Transport and magnetization measurements clearly indicate that spin-polarized quasiparticle injection in $\mathrm{YBa}_{2} \mathrm{Cu}_{3} \mathrm{O}_{7-d} / \mathrm{La}_{0.67} \mathrm{Ca}_{0.33} \mathrm{MnO}_{3}$ (YBCO/LCMO) SL can strongly decrease $T_{c}$ and the critical current $J_{c}$ 10, 11. Particularly interesting is the almost complete spin polarization in LCMO and the low carrier density in YBCO which seems to be suppressed even further in the case of SL, as was shown by optical spectroscopy [12]. The advantage of optical techniques is that one can directly monitor the temperature dependence of the "strength" of the conducting and supercon-

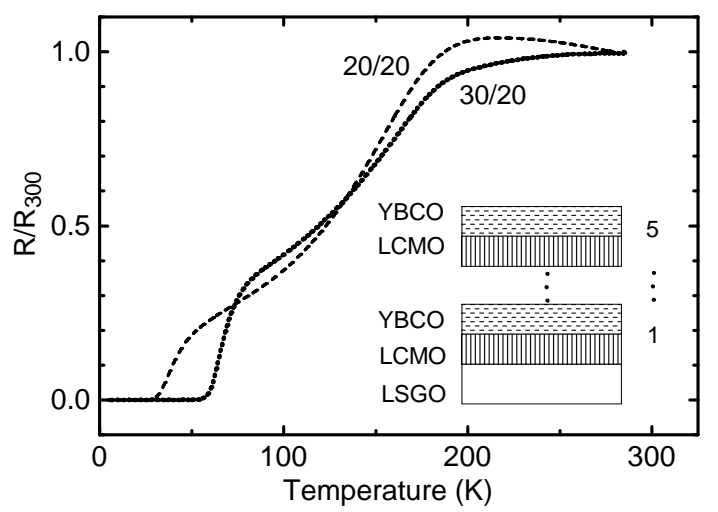

FIG. 1: Temperature dependence of normalized dcresistivities of two superlattices deposited on a $\mathrm{LaSrGaO}_{4}$ substrate, each consisting of five periods of $20 \mathrm{~nm}$ thick $\mathrm{La}_{0.67} \mathrm{Ca}_{0 .}{ }_{33} \mathrm{MnO}_{3}$ layer and 20 or $30 \mathrm{~nm} \mathrm{YBa}_{2} \mathrm{Cu}_{3} \mathrm{O}_{7-d}$ layer, as shown in the inset.

ducting condensates expressed by the spectral weight, i.e. the area under the dynamical conductivity spectrum. Up to now, however, optical measurements failed to even detect a sign of (weakened) superconductivity in SL. In this paper, we report on the first measurements of optical properties of $\mathrm{YBCO} / \mathrm{LCMO}$ superlattices at the radiation quantum energies low enough to allow for observation of SC condensate response. We find strongly reduced SC superfluid density and an enhanced London penetration depth in the superlattices and ascribe it to proximity effects.

The samples are fabricated by pulsed laser deposition techniques on $\mathrm{LaSrGaO}_{4}$ (LSGO) (001) substrates which has low dielectric losses $\left(\tan \delta=5 \times 10^{-5}\right)$ in the used frequency range and a favorable in-plane lattice match between LSGO $(a=b=0.3840 \mathrm{~nm})$ and YBCO $(a=0.3836 \mathrm{~nm}, b=0.3883 \mathrm{~nm})$. The growth conditions of the $c$-axis oriented YBCO and LCMO layers were optimized in the way to achieve optimal superconductivity and ferromagnetism. Detailed information about the fabrication of SLs can be found in Ref. [8]. Alternating 
TABLE I: Parameters of superlattices: thicknesses $d_{\mathrm{YBCO}}$ of $\mathrm{YBa}_{2} \mathrm{Cu}_{3} \mathrm{O}_{7-d}$ and $d_{\mathrm{LCMO}}$ of $\mathrm{La}_{0.67} \mathrm{Ca}_{0.33} \mathrm{MnO}_{3}$ films, number of film periods deposited, superconducting transition temperature $T_{c}$, magnetic ordering temperature $T_{\text {mag }}$ determined by maximum of resistivity, London penetration depth $\lambda_{L}$, plasma frequency of superconducting condensate $\omega_{p}^{s} / 2 \pi$, and scattering rate of normal carriers $\gamma$. Also listed are the literature values for single crystal and thin film of $\mathrm{YBa}_{2} \mathrm{Cu}_{3} \mathrm{O}_{7-d}$ for comparison.

\begin{tabular}{cccccccc}
\hline \hline$d_{\text {YBCO }}$ & $d_{\text {LCMO }}$ & periods & $T_{c}$ & $T_{\text {mag }}$ & $\begin{array}{c}\lambda_{L} \\
(5 \mathrm{~K})\end{array}$ & $\begin{array}{c}\omega_{p}^{s} / 2 \pi \\
(5 \mathrm{~K})\end{array}$ & $\begin{array}{c}\gamma \\
(5 \mathrm{~K}) \\
(\mathrm{nm})\end{array}$ \\
\hline 30 & $(\mathrm{~nm})$ & & $(\mathrm{K})$ & $(\mathrm{K})$ & $(\mu \mathrm{m})$ & $\left(\mathrm{cm}^{-1}\right)$ & $\left(\mathrm{cm}^{-1}\right)$ \\
\hline & 20 & 1 & 70 & & 0.9 & 1780 & 12 \\
30 & 20 & 5 & 55 & 150 & 1.9 & 840 & $>200$ \\
20 & 20 & 5 & 25 & 160 & 4.0 & 400 & $>200$ \\
\hline YBCO crystal (Ref. [17]) & 92 & & 0.14 & 11370 & \\
YBCO film (Ref. [18]) & 90 & & 0.20 & 7960 & \\
\hline \hline
\end{tabular}

LCMO and YBCO layers (five of each) are deposited as sketched in the inset of Fig. 1 For all SLs used, the thickness $d$ of the LCMO layers is kept at $20 \mathrm{~nm}$, while the thickness of the YBCO layers changes from $20 \mathrm{~nm}$ to $30 \mathrm{~nm}$. We have studied two superlattices, which will be referred to as $20 / 20$ and $30 / 20$ - the ratio of thicknesses of the YBCO to the LCMO layers. Parameters of the samples used in our study are listed in Table प Only the corresponding $(00 h)$ peaks are observed in Xray diffraction $\theta / 2$ measurements for YBCO, LCMO, and the LSGO substrate. The SC transition temperature $T_{c}$ and the magnetic transition temperature $T_{\text {mag }}$ are determined by dc-resistance $R(T)$ and SQUID magnetization measurements, respectively (Fig. (1); the values are close to what we have obtained for SLs grown on $\mathrm{SrTiO}_{3}$ substrate [8, 12. In addition, single LCMO film $(d=20 \mathrm{~nm})$ and single YBCO film $(d=30 \mathrm{~nm})$ were also prepared on LSGO substrates so that we could study optical properties of thin YBCO and LSCO films not incorporated in the SL. For a single thin YBCO film of $30 \mathrm{~nm}$ we find a reduced SC transition temperature of approximately 70 to $80 \mathrm{~K}$.

Optical measurements were done in transmission mode at the lower end of the far-infrared spectral range $(6$ - $30 \mathrm{~cm}^{-1}$ ) at temperatures $5 \mathrm{~K}<T<300 \mathrm{~K}$, with the help of the millimeter-submillimeter coherentsource spectrometer utilizing backward-wave oscillators as monochromatic frequency-tunable radiation sources, as described elsewhere in detail [13]. In a Mach-Zehnder arrangement we measure the transmission coefficient $T r$ and the phase shift $\varphi_{t}$ of the radiation passing through the plane-parallel substrate with the SL on it. Spectra of both, conductivity $\sigma(\omega)$ and dielectric permittivity $\epsilon(\omega)$, of single films or of composite SL are obtained directly (no Kramers-Kronig relations used) from $\operatorname{Tr}$ and $\varphi_{t}$ on the basis of the general Fresnel expressions for the layered system [14] (the dielectric parameters of the substrate are measured beforehand). In these measurements the

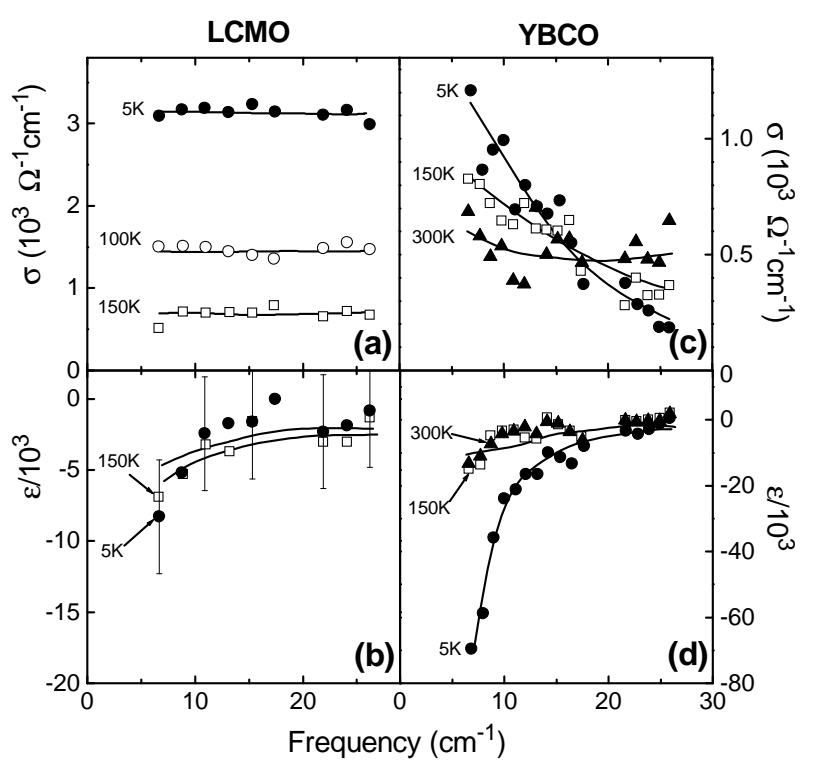

FIG. 2: Frequency dependence of conductivity $\sigma(\omega)$ and dielectric permittivity $\epsilon(\omega)$ of (a and b) single $\mathrm{La}_{0.67} \mathrm{Ca}_{0.33} \mathrm{MnO}_{3}$ (thickness $d=20 \mathrm{~nm}$ ) and (c and d) single $\mathrm{YBa}_{2} \mathrm{Cu}_{3} \mathrm{O}_{7-d}(d=30 \mathrm{~nm})$ films on a $\mathrm{LaSrGaO}_{4}$ substrate at different temperatures as indicated. The lines are guides to the eye.

probing radiation passes through the superlattice which is considered as a single layer and whose effective optical properties are thus averaged over those of SC and FM layers composing it, in conditions when the radiation wavelength is much larger compared to the layer thickness; we note that this transmission technique is thus more sensitive to the optical properties of the films when compared to the optical reflectivity techniques.

Fig. 2 shows the frequency dependence of the conductivity $\sigma(\omega)$ and dielectric permittivity $\epsilon(\omega)$ of single LCMO and YBCO films recorded at different temperatures. For the LCMO film, basically no frequency dependence is observed for $\sigma$ whose value increases at lower temperatures, the spectra of $\epsilon$ show a slight increase to high frequencies and do not significantly depend on the temperature - both observations indicate good metallic (Drude-like) properties. In the case of YBCO, displayed in Fig. 2r and d, both $\sigma$ and $\epsilon$ are frequency independent at high temperatures (metallic behavior) but reveal a pronounced dispersion at $5 \mathrm{~K}$. A strong increase of $\sigma$ is seen towards the lowest frequencies, similar to that observed in all high-Tc's and connected to uncondensed (normal) carriers [15, 16]; such dispersion leads to a peak in the temperature dependence of the conductivity, in case of our film - around $50 \mathrm{~K}$ for the frequency of $25 \mathrm{~cm}^{-1}$. The dielectric permittivity $\epsilon$ shows in the SC state a divergent dispersion $\epsilon \propto-(1 / \omega)^{2}$ which represents the dielectric response of the zero-frequency deltafunction in the conductivity spectrum, responsible for the infinite DC conductivity 14. Both behaviors of $\sigma$ and $\epsilon$ of the YBCO film clearly display the appearance of a 




FIG. 3: Frequency dependence of (a) conductivity $\sigma(\omega)$ and (b) dielectric permittivity $\epsilon(\omega)$ of $\mathrm{YBa}_{2} \mathrm{Cu}_{3} \mathrm{O}_{7-d}$ / $\mathrm{La}_{0.67} \mathrm{Ca}_{0.33} \mathrm{MnO}_{3}$ superlattices (five periods) with $20 \mathrm{~nm}$ thickness of the LCMO film and different thickneses of the YBCO film (20 and $30 \mathrm{~nm}$, denoted as $20 / 20$ and $30 / 20$, respectively), shown for the temperature of $T=5 \mathrm{~K}$. The solid lines are guides to the eye.

SC state. We have analyzed the $5 \mathrm{~K}$ spectra of $\mathrm{YBCO}$ film modeling the response of the condensed SC carriers as $\epsilon_{s}(\omega)=-\left(\omega_{p}^{s} / \omega\right)^{2}$ and a Drude expressions [14] for $\sigma(\omega)$ and $\epsilon(\omega)$ for uncondensed carriers contribution; the results are listed in Table \ For the plasma frequency of the superconducting carriers at $T=5 \mathrm{~K}$ we obtain $\omega_{p}^{s} / 2 \pi=1780 \mathrm{~cm}^{-1}$ from which we get the value of the London penetration depth $\lambda_{L}=c / \omega_{p}^{s}=0.9 \mu \mathrm{m}$ ( $c$ is the velocity of light). Both values are significantly different from those obtained for single crystals and thicker films [17, 18]: the value of $\lambda_{L}$ is strongly enhanced and $\omega_{p}^{s}$ decreased, probably due to the effect of the film thickness, which will be discussed in a subsequent publication.

We now turn to the dielectric responses of the superlattices for which the frequency and temperature dependences are shown in Figs. 3 and 4 . Similar to the single YBCO film, the dielectric permittivity spectra of SLs also reveal a signature of a superconducting response (Fig. 31) - a diverging behavior at the lowest temperature, $\epsilon(\omega) \propto-(1 / \omega)^{2}$. At the same time the absolute values of $\epsilon$, which represent a measure of the SC condensate density, are noticeably, by 6 to 10 times, reduced compared to a single YBCO film. This means that while the superconductivity is still maintained in SLs, its "strength" is strongly suppressed. By fitting the spectra of $\sigma(\omega)$ and $\epsilon(\omega)$ for a SLs as described above we obtain the plasma frequencies of the SC condensate and the London penetration depths, the values given in Table \ 19. We find a strong decrease of the SC carriers density $\left(\omega_{p}^{s}\right)$ and a correspondent increase of $\lambda_{L}$ when going from the single YBCO film to the $30 / 20$ and further to the $20 / 20$ superlattice.

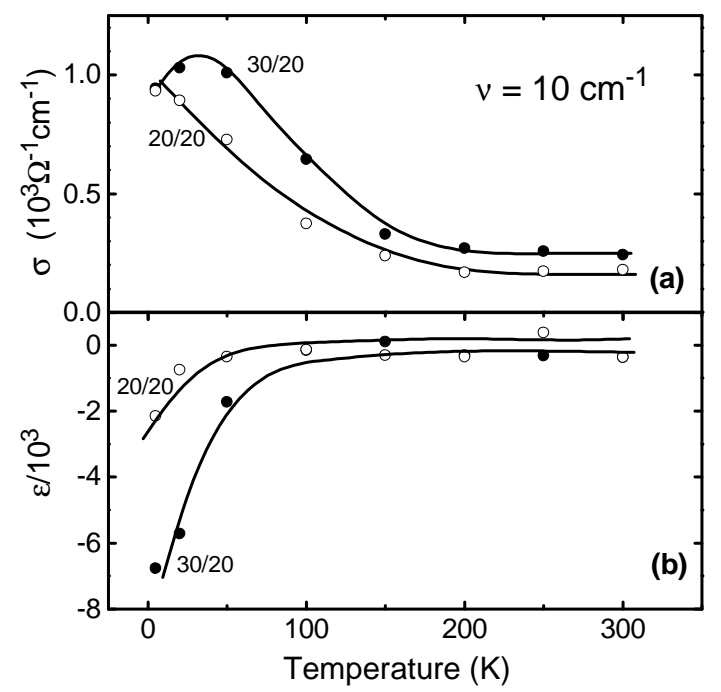

FIG. 4: Temperature dependence of (a) conductivity $\sigma(T)$ and (b) dielectric permittivity $\epsilon(T)$ of $\mathrm{YBa}_{2} \mathrm{Cu}_{3} \mathrm{O}_{7-d} /$ $\mathrm{La}_{0.67} \mathrm{Ca}_{0.33} \mathrm{MnO}_{3}$ superlattices (five periods) with $20 \mathrm{~nm}$ thickness of the LCMO film and different thickness of the YBCO film (20 and $30 \mathrm{~nm}$, denoted as 20/20 and 30/20, respectively). The data are shown for one particular frequency $\nu=10 \mathrm{~cm}^{-1}$. The solid lines are guides to the eye.

The conductivity spectra of SLs also show behaviors different from that in the single YBCO film. The absolute values of the conductivities for the $20 / 20$ and $30 / 20$ SLs are reduced and no significant dispersion is observed, both indicating an increase of the scattering rate for the normal (uncondensed) carriers.

The observed suppression of SC in the superlattices cannot be caused by such effects as incomplete oxygenation or surface disorder, as was shown in [8] and [20]. We also note that we were not able to fit the measured spectra of transmission coefficient and phase shift by considering the SL as consisting of $5 \mathrm{YBCO}$ and 5 LCMO layers each having properties of single YBCO and LCMO films. This means that these films, when incorporated in a SL, cannot be considered as independent layers. We thus conclude that it is the interaction of FM and SC condensates that is responsible for the strong reduction of the observed SC carrier density. Our findings are in accordance with estimations of the spin diffusion length of approximetely $30 \mathrm{~nm} \mathrm{[21]}$.

In the study of impurity doped YBCO, the normalized critical temperature $T_{c} / T_{c 0}$ ( $T_{c 0}$ corresponds to the transition temperature of a high quality YBCO) is found to show the dependence on the normalized zero temperature superfluid density $n_{s} / n_{0}$ [22, 23] which is quite different from that predicted by a standard Abrikosov-Gor'kov theory 24]. Using $T_{c 0}=92 \mathrm{~K}$ (YBCO single crystal), we found that the ratio $T_{c} / T_{c 0}$ for our superlattices shows behaviors similar to that in $\mathrm{Zn}$ - and Ni-doped YBCO thin films $[24,25]$; those results were explained by a gapless superconductivity. In fact for low- $T_{c} \mathrm{SC} / \mathrm{FM}$ superlattices Sun et al. [25] recently proposed that gapless 
superconductivity appears in both FM and SC regions near the interface. In the superlattices, the influence of the FM layers upon the SC layers is expressed via a strong plane perturbation, magnetic exchange field and proximity effect. As first pointed out by Abrikosov and Gor'kov 24], gapless superconductivity is a common feature in $\mathrm{SC}$ in the presence of strong perturbation. In the gapless region, the electronic properties are drastically modified and lead, for example, to a linearly temperature dependent specific heat, reduced $T_{c}$ and enhanced London penetration depth 27], in accordance with our optical observations.
In summary, from our low-energy optical experiments we find a strong weakening of the superconducting response of superconducting/ferromagnetic YBCO/LCMO superlattices when the thickness of the YBCO SC layer in the superlattice decreases: considering the superlattice as an effective medium we find a strong decrease of the effective density of the superconducting condensate and correspondent enhancement of the London penetration depth. These results indicate a strong suppression of superconductivity in $\mathrm{YBCO} / \mathrm{LCMO}$ superlattices which can be attributed to the pair-breaking effect of the ferromagnetic layers.
[†] Permanent address: General Physics Institute, Russian Academy of Sciences, Moscow, Russia.

[*] Electronic address: dressel@pi1.physik.uni-stuttgart.de

[1] Ø. Fisher and M. Peter, in: Magnetism, edited by G.T. Rado and H. Suhl (Academic Press, New York, 1973), Vol. V, p. 327; M.B. Maple and Ø. Fisher, Superconductivity and Magnetism, (Springer-Verlag, Berlin, 1982)

[2] P. Fulde and A. Ferrell, Phys. Rev. 135, A550 (1964); A. Larkin and Y. Ovchinnikov, Sov. Phys. JETP 20, 762 (1965).

[3] C.L. Chien and D.H. Reich, J. Magn. Magn. Mater. 200, $83(1999)$

[4] Yu.A. Izyumov, Yu. N. Proshin, and M.G. Khusainov, Physics Uspekhi 45, 109 (2002).

[5] M. Johnson, J. Supercond. 14, 273 (2001); A.M. Goldman, P.I. Kraus, K. Nikovaev, V. Vas'ko, A. Bhattacharya, and W. Cooley, J. Supercond. 14, 283 (2001)

[6] G. Jakob, V.V. Moshchalkov, and Y. Bruynseraede, Appl. Phys. Lett. 66, 2546 (1995).

[7] P. Przysłupski, T. Nishizaki, N. Kobayashi, S. Koleśnik, T. Skośkiewicz, and E. Dynowska, Physica B 259-261, 820 (1999)

[8] H.-U. Habermeier, G. Cristiani, R.K. Kremer, O. Lebedev, and G. van Tendeloo, Physica C 364-365, 298 (2001)

[9] O. Pieto et al., J. Appl. Phys. 89, 8026 (2001).

[10] V.A. Vas'ko et al., Phys. Rev. Lett. 78, 1134 (1997); A.M. Goldman, V. Vas'ko, P. Kraus, K. Nikolaev, and V.A. Larkin, J. Magn. Magn. Mater. 200, 69 (1999).

[11] N.-C. Yeh, R. P. Vasquez, C. C. Fu, A. V. Samoilov, Y. Li, and K. Vakili, Phys. Rev. B 60, 10522 (1999).

[12] T. Holden, et al., cond-mat/0303284

[13] Kozlov G.V. and Volkov A.A., Millimeter and Submillimeter Wave Spectroscopy of Solids, ed. by Grüner G. (Springer, Berlin, 1998), p. 51.
[14] M. Dressel and G. Grüner, Electrodynamics of Solids (Cambridge University Press, Cambridge, 2002).

[15] D.B. Tanner and T. Timusk, in: Physical Properties of High Temperature Superconductors Vol. III, edited by D. M. Ginsberg (World Scientific, Singapore, 1992), p. 363.

[16] D.A. Bonn and W. N. Hardy: in Physical Properties of High Temperature Superconductors Vol. V, edited by D.M. Ginsberg (World Scientific, Singapore, 1996), p. 7.

[17] W.N. Hardy et al., Phys. Rev. Lett. 70,3999 (1993).

[18] P. Chaudhari, R. H. Koch, R. B. Laibowitz, T. R. McGuire and R. J. Gambino, Phys. Rev. Lett. 58, 2684 (1987); H.-U. Habermeier, G. Beddies, B. Leibold, G. Lu, and G. Wagner, Physica C 180, 17S (1991); S. Djordjevic, Eur. Phys. J. B 5, 847 (1998); L. A. de Vaulchier et al., Phys. Rev. B 52, 564 (1995).

[19] Although there is a clear tendency of the values with increasing thickness $d_{\mathrm{YBCO}}$, a spread in the sample quality, in particular the interfaces, leads to some scattering of the data.

[20] Z. Sefrioui et al., Appl. Phys. Lett. 81, 4568 (2002).

[21] S. Soltan, J. Albrecht, G. Cristiani, and H.-U. Habermeier, Europhys. Lett. 63,881 (2003).

[22] E.R. Ulm, J. Kim, T.R. Lemberger, S.R. Foltyn, and X. Wu, Phys. Rev. B 51, 9193 (1995)

[23] M. Franz, C. Kallin, A.J. Berlinsky, and M.I. Salkola, Phys. Rev. B 56, 7882 (1997).

[24] A.A. Abikosov and L. P. Gor'kov, Soviet Phys. JETP 12, 1243 (1961).

[25] G. Sun, D.Y. Xing, J. Dong and M. Liu, Phys. Rev. B 65, 174508 (2002).

[26] D.H. Lowndes et al., Phys. Rev. Lett. 65, 1160 (1990).

[27] K. Maki, in: Superconductivity, Vol. 2, edited by R.D. Parks (Marcel Dekker, Inc., New York, 1969), p. 1035. 